\title{
On the continuous solutions of a non-linear functional equation of the first order
}

\author{
by Karol BaRon (Katowice)
}

\begin{abstract}
Some theorems abont the existence, uniqueness and properties of the continuous solution of the functional equation

$$
\varphi(x)=h(x, \varphi[f(x)]),
$$
\end{abstract}

where $\varphi$ is the unknown function, are given, under the hypothesis of the existence of semicontinuous solutions of the functional inequalities

and

$$
\varphi(x) \leqslant h(x, \varphi[f(x)])
$$

$$
h(x, \varphi[f(x)])<\varphi(x) .
$$

In the present paper we are concerned with the existence, uniqueness and some properties of the continuous solutions of the functional equation

$$
\varphi(x)=h(x, \varphi[f(x)]),
$$

where $\varphi$ is the unknown function.

We assume that

(i) The real function $f$ is defined and continuous in an interval $I$ and, for a $\xi \in I$, it fulfils the inequalities

$$
0<\frac{f(x)-\xi}{x-\xi}<1 \quad \text { for } \quad x \in I, x \neq \xi
$$

(ii) $h$ is a real function defined and continuous in a set $\Omega \subset R^{2}$ containing the point $(\xi, \eta)$, where $\eta$ is a solution of the equation

$$
\eta=h(\xi, \eta) \text {. }
$$

Moreover, in a neighbourhood of $(\xi, \eta) h$ fulfils a Lipschitz condition with respect to the second variable

$$
\left|h\left(x, y_{1}\right)-h\left(x, y_{2}\right)\right| \leqslant \gamma(x)\left|y_{1}-y_{2}\right|, \quad\left(x, y_{i}\right) \in U \cap \Omega ; i=1,2,
$$

where

$$
U=U_{1} \times U_{2}, \quad U_{1}=\{x \in R:|x-\xi| \leqslant c\}, \quad U_{2}=\{y \in R:|y-\eta| \leqslant d\} .
$$

and $c, d$ are positive numbers. 
For a subinterval $J$ of $I$ we denote by $\Phi(J)$ the class of all real functions $\varphi$ defined on $J$ and fulfilling the condition $\varphi[f(x)] \epsilon \Omega_{x}$ for $x \in J$, where $\Omega_{x}$ denotes the $x$-section of $\Omega$ :

$$
\Omega_{x}=\{y \in R:(x, y) \in \Omega\},
$$

(cf. [2], p. 68).

Our next assumptions read as follows:

(iii) The functional inequality

$$
\varphi(x) \leqslant \hbar(x, \varphi[f(x)])
$$

has in the class $\Phi(I)$ a lower semicontinuous solution $\varphi_{1}^{*}$, whereas the inequality

$$
h(x, \varphi[f(x)]) \leqslant \varphi(x),
$$

has in this class an upper semicontinuous solution $\varphi_{2}^{*}$. Furthermore $\varphi_{1}^{*}(x)$ $\leqslant \varphi_{2}^{*}(x)$ for $x \in I$;

(iv) For every fixed $x \in I$ the set $\Omega_{x}$ is an interval, $h\left(f(x), \Omega_{f(x)}\right) \subset \Omega_{x}$ and $h$ is an increasing function with respect to the second variable in the interval $I(x)=\left\langle\varphi_{1}^{*}[f(x)], \varphi_{2}^{*}[f(x)]\right\rangle$.

Put

$$
G_{n}(x)=\prod_{i=0}^{n-1} \gamma\left[f^{i}(x)\right], \quad n=1,2, \ldots
$$

We have the following

Theorem 1. Suppose that hypotheses (i)-(iv) are fulfilled. If equation (2) has in the interval $I(\xi)$ exactly one solution $\eta$ and the sequence $\left\{G_{n}\right\}$ defined by (5) is bounded in a vicinity of the point $\xi$ :

$$
G_{n}(x) \leqslant M(x), \quad x \in I \cap\langle\xi-\delta, \xi+\delta\rangle, x \neq \xi ; n=1,2, \ldots,
$$

then equation (1) has exactly one solution $\varphi \in \Phi(I)$ continuous at the point $\xi$ and such that $\varphi(\xi)=\eta$. This solution is a continuous function, fulfils the condition

$$
\varphi_{1}^{*}(x) \leqslant \varphi(x) \leqslant \varphi_{2}^{*}(x), \quad x \in I,
$$

and is given by the formula

$$
\varphi(x)=\lim _{n \rightarrow \infty} \varphi_{n}(x),
$$

where

$$
\varphi_{n+1}(x)=h\left(x, \varphi_{n}[f(x)]\right), \quad n=0,1,2, \ldots
$$

and $\varphi_{0}$ is an arbitrary function belonging to $\Phi(I)$, continuous at the point $\xi$, and such that $\varphi_{0}(\xi)=\eta$. 
Proof. Let $\Phi_{1}$ be the class of all lower semicontinuous functions $\varphi$ belonging to $\Phi\left(I_{0}\right)$ and fulfilling the condition

$$
F_{1}^{*}(x) \leqslant \varphi(x) \leqslant \varphi_{2}^{*}(x) ; \quad x \in I_{0},
$$

where $I_{0}=U_{1} \cap\langle\xi-\delta, \xi+\delta\rangle \cap I$; and similarly, let $\Phi_{2}$ be the class of all upper semicontinuous functions $q$ belonging to $\Phi\left(I_{0}\right)$ and fulfilling condition (9). Put

$$
\begin{aligned}
\varphi_{i, 0}(x)=\varphi_{i}^{*}(x), \quad \varphi_{i, n+1}(x)=h\left(x, \varphi_{i, n}[f(x)]\right), \\
\quad x \in I_{0} ; i=1,2 ; n=0,1,2, \ldots
\end{aligned}
$$

It follows from the continuity of the functions $f$ and $h$ and from hypotheses (i), (iii) and (iv) that

$$
\varphi_{i, n} \in \Phi_{i}, \quad i=1,2 ; n=0,1,2, \ldots
$$

and

$$
\varphi_{1, n}(x) \leqslant \varphi_{1, n+1}(x), \quad \varphi_{2, n+1}(x) \leqslant \varphi_{2, n}(x), \quad x \in I_{0} ; n=0,1,2, \ldots
$$

Hence the sequences $\left\{p_{i, n}\right\}, i=1,2$, are convergent. Let

$$
\varphi_{i}(x)=\lim _{n \rightarrow \infty} \varphi_{i, n}(x), \quad x \in I_{0} ; i=1,2 .
$$

On account of (11), (12) and (13)

$$
\varphi_{i} \in \Phi_{i}, \quad i=1,2 \text {. }
$$

Moreover, by (10) and (13) we have

$$
\varphi_{i}(x)=\hbar\left(x, \varphi_{i}[f(x)]\right), \quad x \in I_{0} ; i=1,2 .
$$

Putting $x=\xi$ in (15) and making use of the facts that $f(\xi)=\xi$ and that $\eta$ is the unique solution of equation $(2)$ in $I(\xi)$, we obtain

$$
\varphi_{i}(\xi)=\eta, \quad i=1,2 .
$$

We shall show that

$$
\limsup _{x \rightarrow \xi} \varphi_{1}(x) \leqslant \eta, \quad \liminf _{x \rightarrow \xi} \varphi_{2}(x) \geqslant \eta .
$$

Indeed, write $\limsup _{x \rightarrow \xi} p_{1}(x)=a_{0}$. Evidently $a_{0} \in I(\xi)$. There exists a sequence $\left\{x_{n}\right\}, x_{n} \in I_{0}$, such that $\lim _{n \rightarrow \infty} x_{n}=\xi$ and $\lim _{n \rightarrow \infty} \varphi_{1}\left(x_{n}\right)=a_{0}$. From the sequence $\left\{\varphi_{1}\left[f\left(x_{n}\right)\right]\right\}$ we can choose a convergent subsequence $\left\{\varphi_{1}\left[f\left(x_{n_{k}}\right)\right]\right\}$. Since $\lim _{k \rightarrow \infty} f\left(x_{n_{k}}\right)=\xi$, we have $\lim _{k \rightarrow \infty} \varphi_{1}\left[f\left(x_{n_{k}}\right)\right]=b \leqslant a_{0}, b \in I(\xi)$. Hence and from (15) we obtain $a_{0}=h(\xi, b) \leqslant h\left(\xi, a_{0}\right)$. Let $a_{n+1}=h\left(\xi, a_{n}\right), n=0,1,2, \ldots$ The sequence $\left\{a_{n}\right\}$ is increasing and hounded from above $\left(a_{n} \in I(\xi)\right.$, $n=0,1,2, \ldots)$, and so it is convergent. Its limit belongs to $I(\xi)$ and fulfils equation (2) and hence it must be equal to $\eta$. By the monotonicity 
of $\left\{a_{n}\right\}$ we have $a_{0} \leqslant \eta$. Sinilarly we can prove the second inequality in (17). Thus in view of (14), (16) and (17) we have the continuity of $q_{i}$, for $i=1,2$, at the point $\xi$. A similar argument as in proof of Theorem 1 in [1] shows that

$$
\varphi_{1}(x)=p_{2}(x), \quad x \in I_{0}, x \neq \xi .
$$

Hence and from (16) $\varphi_{1}=\varphi_{2}=\bar{\varphi}$. By (14) $\bar{\varphi} \epsilon \Phi_{1} \cap \Phi_{2}$ turns out to be a continuous solution of equation (1) in $I_{0}$. This solution can be uniquely extended onto the whole interval $I$ and the extension $\varphi$ is continuous ([2], p. 70, Theorem 3.2). We shall show that $\varphi$ fulfils (7). For the indirect proof suppose that there is an $x_{0} \in I$ such that the condition

$$
p_{1}^{*}\left(x_{0}\right) \leqslant \varphi\left(x_{0}\right) \leqslant \varphi_{2}^{*}\left(x_{0}\right)
$$

is not fulfilled. Since $\lim _{n \rightarrow \infty} f^{n}\left(x_{0}\right)=\xi$, there is a non-negative integer $n$ such that the condition

$$
\varphi_{1}^{*}\left[f^{n}\left(x_{0}\right)\right] \leqslant p\left[f^{n}\left(x_{0}\right)\right] \leqslant \varphi_{2}^{*}\left[f^{n}\left(x_{0}\right)\right]
$$

is not fulfilled, whereas the condition

$$
\varphi_{1}^{*}\left[f^{n+1}\left(x_{0}\right)\right] \leqslant \varphi\left[f^{n+-1}\left(x_{0}\right)\right] \leqslant \varphi_{2}^{*}\left[f^{n+1}\left(x_{0}\right)\right]
$$

is fulfilled. Hence, and from hypotheses (iii) and (iv) we obtain

$$
\begin{aligned}
{\varphi_{1}^{*}}_{1}^{*}\left[f^{n}\left(x_{0}\right)\right] & \leqslant h\left(f^{n}\left(x_{0}\right), \phi_{1}^{*}\left[f^{n+1}\left(x_{0}\right)\right]\right) \leqslant h\left(f^{n}\left(x_{0}\right), \varphi\left[f^{n+1}\left(x_{0}\right)\right]\right) \\
& \leqslant h\left(f^{n}\left(x_{0}\right), \varphi_{2}^{*}\left[f^{n+1}\left(x_{0}\right)\right]\right) \leqslant \varphi_{2}^{*}\left[f^{n}\left(x_{0}\right)\right] .
\end{aligned}
$$

This together with (1) contradicts our assumption. Recalling the proofs of Theorems 2 and 1 in [1] and the fact that $\varphi(\xi)=\varphi_{n}(\xi)=\eta$ for $n=$ $0,1,2, \ldots$, we get formula (8) and the required uniqueness, respectively.

The next two theorems say something about the properties of the solutions obtained.

THeOREar 2. Suppose that the hypotheses of Theorem 1 aie filfilled. If $f$ is increasing and $h$ is increasing with respect to each variable in the set $\bigcup\{\{x\} \times I(x): x \in I\}$ and, moreaver, $\varphi_{1}^{*}$ or $\varphi_{2}^{*}$ is increasing, then the solution obtained in Theorem 1 is an increasing function. .

THEOREM 3. Suppose that the hypotheses of Theorem 1 are fulfilled. If $f$ is increasing and convex and $h$ is increasing with sespect to each variable and convex in the set $\bigcup\{\{x\} \times I(x): x \in I\}$, whereas $\varphi_{1}^{*}$ is increasing and convex and $p_{2}^{*}$ is decreasing and concave, then the solution obtained in Theorem 1 is an increasing and convex function.

In fact, in the case of Theorem 2 , for every $n=0,1,2, \ldots, \varphi_{1, n}$ defined by (10) is an increasing function or for every $n=0,1,2, \ldots, \varphi_{\mathrm{a}, n}$ defined by (10) is an increasing function. In the case of Theorem 3 , for every $n=0,1,2, \ldots, \varphi_{1, n}$ is an increasing and convex function. Fur- 
thermore, the extension of an increasing solution is increasing and the extension of an increasing and convex solution is increasing and convex (cf. [4]).

Now we shall show that the above theorems imply the following results.

THEOREMI 4. Let hypotheses (i), (ii) and condition (6) be fufilled and suppose that $\Omega$ is an open set ( $\left.{ }^{1}\right)$. If , for every fixed $x \in D_{1}$, $h$ is an increasing function with respect to the second variable in $U_{2}$ and

$$
|h(\xi, y)-\eta|<|y-\eta|, \quad y \in U_{\mathrm{a}}, y \neq \eta,
$$

then in a neighbourhood of $\xi$ there exists a unique solution $\varphi$ of equation (1) which is continuous at $\xi$ and such that $\varphi(\xi)=\eta$. This solution is a continuous function.

ThEOREM 5. S'uppose that the hypotheses of Theorem 4 are fulfilled. If $f$ is increasing [and convex] in $I$ and $h$ is increasing with respect to each variable [and convex] in $0 \cap \Omega$, then the solution obtained in Theorem 4 is an increasing [and convex] function.

Indeed, it follows from (18) and from the continuity of $h$ that there exist positive numbers $c_{0}, d_{0}$ such that $I_{0} \subset D_{1} \cap I, I_{0} \times\left\langle\eta-d_{0} ; \eta+d_{0}\right\rangle$ $\subset U \cap \Omega$ and $|h(x, y)-\eta| \leqslant d_{0}$ for $(x, y) \in I_{0} \times\left\langle\eta-d_{0}, \eta+d_{0}\right\rangle$, where $I_{0}$ $=\left\langle\xi, \xi+c_{0}\right\rangle$ if $\xi$ is the left endpoint of the interval $I, I_{0}=\left\langle\xi-c_{0}, \xi+c_{0}\right\rangle$ if $\xi$ is an interior point of $I$, and $I_{0}=\left\langle\xi-c_{0}, \xi\right\rangle$ if $\xi$ is the right endpoint of $I$. Hence, the functions $\varphi_{i}^{*}, i=1,2$, defined by

$$
\varphi_{1}^{*}(x)=\eta-d_{0}, \quad \varphi_{2}^{*}(x)=\eta+d_{0} ; \quad \text { ir } \in I_{0}
$$

are continuous solutions of inequalities (3) and (4), respectively, and $\eta$ is the unique solution of equation (2) in the interval $\left\langle\eta-d_{0}, \eta+d_{0}\right\rangle$.

\section{References}

[1] D. Czaja-Pośpiech and M. Kuczma, Continuous solutions of some functional equations in the indeterminate ease, Ann. Polon. Matl. 24 (1970), p. 9-20.

[2] M. Kuczma, Functional equations in a single variable, Monografie Mat. 46, Warszatwa 1968.

[3] - Special solutions of a functional equation, Ann. Polon. Math. 27(1972), p. 29-32.

[4] J. Afatkowski, On some properties of solutions of a funclional equation, Prace Naukowe Eniwersytetu Sląskiego w Katowicach, Prace Mat. 1 (1969), p. 79-82.

Reṣu par la Rédaction le 20. 1. 1972

(1) Instead of this assumption we may require that $\Omega$ should contain a rectangle $Q$ (possibly closed) such that $(\xi, \eta) \in Q$ and $(x, h(x, y)) \in Q$ for $(x, y) \in Q$ (cf. [3]). 